\title{
Análise de Custo-efetividade do rastreamento do câncer de mama com mamografia convencional, digital e ressonância
}

\author{
Analysis of Cost-effectiveness of screening for breast cancer \\ with conventional mammography, digital \\ and magnetic resonance imaging
}

\author{
Antonio Augusto de Freitas Peregrino ${ }^{1}$ \\ Cid Manso de Mello Vianna ${ }^{2}$ \\ Carlos Eduardo Veloso de Almeida ${ }^{3}$ \\ Gabriela Bittencourt Gonzáles ${ }^{4}$ \\ Samara Cristina Ferreira Machado ${ }^{5}$ \\ Frances Valéria Costa e Silva ${ }^{6}$ \\ Marcus Paulo da Silva Rodrigues ${ }^{2}$
}

\footnotetext{
${ }^{1}$ Laboratório de Ciências Radiológicas, Universidade do Estado do Rio de Janeiro. Rua São Francisco Xavier 524/Prédio Haroldo Lisboa da Cunha/136/térreo, Maracanã. 20550-900 Rio de Janeiro RJ.

antoniop@uerj.br ${ }^{2}$ Instituto de Medicina Social, Universidade do Estado do Rio de Janeiro ${ }^{3}$ Laboratório de Ciências Radiológicas, Universidade do Estado do Rio de Janeiro ${ }^{4}$ Departamento de Saúde e Sociedade, Universidade Federal Fluminense ${ }^{5}$ Instituto de Biologia, Universidade Federal Fluminense

${ }^{6}$ Faculdade de Enfermagem, Universidade do Estado do Rio de Janeiro
}

\begin{abstract}
A cost-effectiveness analysis was conducted in screening for breast cancer. The use of conventional mammography, digital and magnetic resonance imaging were compared with natural disease history as a baseline. A Markov model projected breast cancer in a group of 100,000 women for a 30 year period, with screening every two years. Four distinct scenarios were modeled: (1) the natural history of breast cancer, as a baseline, (2) conventional film mammography, (3) digital mammography and (4) magnetic resonance imaging. The costs of the scenarios modeled ranged from $R \$ 194.216,68$ for natural histo$r y$, to $R \$ 48.614 .338,31$, for screening with magnetic resonance imaging. The difference in effectiveness between the interventions ranged from 300 to 78.000 years of life gained in the cohort. The ratio of incremental cost-effectiveness in terms of cost per life-year gains, conventional mammographic screening has produced an extra year for $R \$ 13.573,07$. The ICER of magnetic resonance imaging was $R \$ 2.904 .328,88$, compared to no screening. In conclusion, it is more costeffective to perform the screening with conventional mammography than other technological interventions.
\end{abstract}

Key words Cost-effectiveness, Markov model, Mammography
Resumo O objetivo deste trabalho foi realizar análise de custo efetividade da intervenção das mamografias convencional e digital e da ressonância magnética no rastreamento de câncer de mama, comparando com o não rastreamento. Foi construído um modelo markoviano, numa uma coorte hipotética de 100 mil mulheres com rastreamento bianual, cuja linha de base é a história natural da doença. Modelaram-se quatro cenários distintos: (1) a história natural do câncer de mama como linha de base; (2) mamografia com filme convencional; (3) mamografia digital e (4) e ressonância magnética. Os custos dos cenários modelados variaram desde $R \$ 194.216,68$ para a história natural, até $R \$ 48.614 .338,31$ para o rastreamento com ressonância magnética. As diferenças de efetividade entre as intervenções variaram de 300 até 78.000 anos de vida ganhos, na coorte de 100 mil mulheres. Em relação à Razão de Custo-Efetividade Incremental, em termos de custo por ano de vida ganhos, a estratégia do rastreamento mamográfico convencional produziu um ano extra por $R \$ 13.573,07$. A Razão de Custo Efetividade Incremental (ICER) da ressonância magnética foi de $R \$ 2.904 .328,88$ em relação ao não rastreamento. O estudo mostrou que é mais custo-efetivo realizar o rastreamento com a mamografia convencional do que as outras tecnologias de intervenção.

Palavras-chave Custo-efetividade, Cadeia de Markov, Mamografia, Câncer de mama 


\section{Introdução}

A incidência mundial de câncer de mama está projetada para 1,45 milhões de casos novos em 2010. O câncer de mama é o mais prevalente hoje no mundo devido à sua alta incidência e ao seu bom prognóstico em estadios iniciais; entretanto, é a causa mais comum de mortes relacionadas às mulheres em nível mundial ${ }^{1}$.

O Instituto Nacional do Câncer (INCA) estima que em 2010, dos prováveis 489.270 casos novos de câncer, 49.240 serão de câncer de mama, gerando um risco de 49 casos novos a cada 100 mil mulheres ${ }^{2}$. Trata-se da patologia maligna mais incidente na população feminina e seu quadro é agravado pelo fato do diagnóstico ainda ser estabelecido, no Brasil, na maioria das vezes, numa fase tardia da doença. Ainda segundo dados do INCA de 1998, neste ano, os diagnósticos ocorriam, na maior parte das vezes, no estadiamento III $(44,8 \%)$ e IV $(16,3 \%)$, enquanto apenas $6,3 \%$ dos diagnósticos davam-se nos estadios 0 e I³.

$\mathrm{O}$ rastreamento mamográfico tem sido uma ferramenta importante no diagnóstico precoce, tendo impacto fundamental na história natural da doença, traduzido na detecção de tumores em estadios iniciais e redução da mortalidade. A intervenção da mamografia tem limitações e riscos, como qualquer outra tecnologia de rastreamento, pois a acurácia não é de $100 \%$, gerando achados falso-positivos e falso-negativos ${ }^{4-6}$.

$\mathrm{Na}$ revisão de literatura realizada por Hider e Nicholas ${ }^{7}$, alguns estudos consideraram que a acurácia do rastreamento mamográfico e o efeito da intervenção na sobrevida aumentam com a idade. Em mulheres entre 50 e 65 anos de idade, o rastreamento mamográfico parece ser mais efetivo, pois reduz a mortalidade em cerca de $20 \%$. No entanto, existe significativa taxa de falso-positivos para a mamografia, com uma contribuição substancial dos custos associados com a intervenção.

Vários estudos de custo-efetividade (6,8-10 $^{6}$ êm sido realizados para o rastreamento de câncer de mama, mas os resultados ainda continuam conflitantes devido à dificuldade de se relacionar os benefícios do rastreamento sistemático aos custos decorrentes dos procedimentos diagnósticos nas mulheres examinadas, para o sistema de saúde, incluindo os custos não monetários.

Novas tecnologias para o rastreamento do câncer de mama têm sido desenvolvidas. A intensidade e a velocidade destas tecnologias e sua incorporação ao sistema de saúde podem causar mudanças significativas, que vão desde os processos diagnósticos e terapêuticos utilizados nes- ta prática até alterações na forma e organização dos serviços, gerando impactos financeiros importantes.

$\mathrm{O}$ advento da mamografia digital é hoje uma realidade, muitas transições de plataformas de mamografia analógicas para digital estão sendo realizados no intuito de aumentar a eficácia deste exame. A mamografia digital tem se mostrado vantajosa em certos grupos de mulheres, tais como aquelas com menos de 50 anos de idade, na pré ou na perimenopausa e com mamas densas e heterogêneas. Isto se deve principalmente pela maior resolução e contraste da imagem ${ }^{11,12}$. Apesar de a imagem digital permitir um armazenamento mais rápido, a aquisição de uma unidade digital pode custar de 1,5 a 4 vezes a mais do que uma unidade de filme ${ }^{13}$. Existem outros fatores que podem contribuir para um maior impacto da mamografia digital, tais como: armazenagem e transmissão de dados, imagens transmitidas para vários médicos sem perda da qualidade, eliminação de artefatos e sujeira dos filmes causados no processamento destes e a capacidade de variação do contraste da imagem. Mas, de maneira geral, a acurácia diagnóstica no rastreamento entre a mamografia digital e filme são similares ${ }^{14,15}$.

Os benefícios da imagem de ressonância magnética (IRM) de mama estão relacionados à sua alta sensibilidade na detecção da doença com a utilização do contraste com gadolíneo. A IRM de mama tem demonstrado ser útil em diferentes situações, a saber: no rastreamento de pacientes com alto risco de câncer de mama, monitoramento de resposta ao tratamento de pacientes submetidas à quimioterapia adjuvante, avaliação de pacientes com adenocarcinoma axilar metastático e tumor primário desconhecido, entre outras coisas ${ }^{16}$. As desvantagens estão relacionadas principalmente a: baixa especificidade do exame, custo cerca de vinte vezes maior do que o filme, longo tempo para realização exame e incapacidade de detectar micro calcificações relacionadas ao carcinoma ductal in situ ${ }^{16,17}$

Este estudo teve como objetivo principal realizar uma análise de custo-efetividade da intervenção das mamografias convencional e digital e da ressonância magnética, no rastreamento de câncer de mama, comparando estas intervenções com o não rastreamento.

\section{Metodologia}

Foi construído um modelo da historia natural da doença através da cadeia de Markov para o cân- 
cer de mama, numa coorte hipotética de 100 mil mulheres que pode ser visualizado na Figura 1.

O modelo foi baseado na participação de $100 \%$ da coorte no rastreamento. No entanto, há possibilidade das mulheres buscarem o médico fora de um programa de rastreamento. De acordo com Hunter et al. ${ }^{17}$, existe uma probabilidade do aparecimento clínico de lesões que poderiam ser detectadas através do autoexame. Foram desenvolvidos quatro cenários distintos, todos eles iniciando os rastreamentos a partir dos 50 anos. O primeiro cenário modelou a his- tória natural do câncer de mama, sem intervenção (baseline). No segundo cenário, foi realizada modelagem da coorte supracitada com a intervenção da mamografia com filme écran ou convencional. O terceiro cenário analisou a intervenção do rastreamento através da mamografia digital e, por fim, foi feita a modelagem com a intervenção da ressonância magnética. A periodicidade do rastreamento foi bianual, de acordo com o Instituto Nacional de Câncer - INCA ${ }^{18}$. A medida de efetividade utilizada neste trabalho foram Anos de Vida Ganhos.

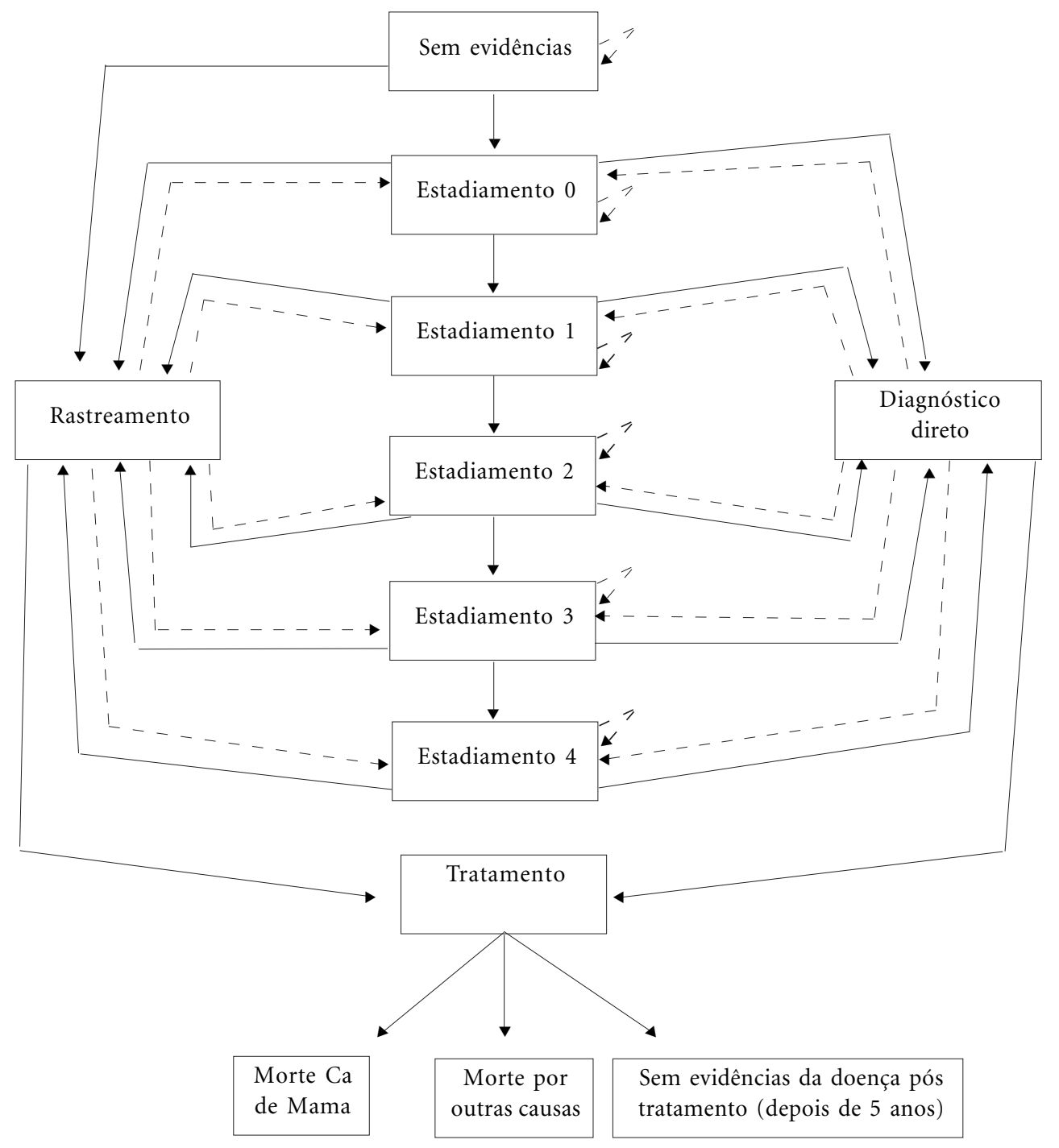

Figura 1. Modelo Teórico do Rastreamento do Câncer de Mama utilizado. Cada flecha representa a probabilidade de um indivíduo que está em um estado X mover-se para um estado Y, Adaptado de: Hunter et al. ${ }^{17}$. 
Tabela 1. Parâmetros utilizados no modelo com seus limites inferiores e superiores: a) probabilidades de transição; b) efetividade da intervenção; c) custos de diagnóstico e d) custos com equipamentos

\begin{tabular}{|c|c|c|}
\hline Parâmetros & Probabilidade anual & Referência \\
\hline a) Probabilidades de transição & $\begin{array}{l}\text { Probabilidade } \\
\text { Inferior - superior }\end{array}$ & \\
\hline $\begin{array}{l}\text { Taxa de Mortalidade (morte por outras causas) / 100.000, } \\
\text { por faixa etária }\end{array}$ & $0,00109-0,49058$ & 17 \\
\hline $\begin{array}{l}\text { Taxa de incidência de câncer de mama por faixa etária/ } \\
100.000\end{array}$ & $0,0000949-0,0044932$ & 18 \\
\hline Prevalência/100.000, por faixa etária & $0,000673-0,056435$ & 2,15 \\
\hline E0 & $0,000662-0,006214$ & \\
\hline E 1 & $0,0000719342-0,002216$ & \\
\hline E2 & $0,00003797-0,000966176$ & \\
\hline E3 & 0,000020878 & \\
\hline $\mathrm{E} 4$ & 0,000365197 & \\
\hline b) Efetividade da Intervenção & Inferior - Superior & \\
\hline Sensibilidade da mamografia por faixa etária & $0,76-0,99$ & 15 \\
\hline Especificidade da mamografia por faixa etária & 0,91 & 15 \\
\hline Taxa de transição de estadio por faixa etária & & 15 \\
\hline Sem evidências - E0 & $0,00065-0,00246$ & \\
\hline E0-E1 & $0,259-0,299$ & \\
\hline E1-E2 & $0,079-0,069$ & \\
\hline E2-E3 & $0,128-0,08$ & \\
\hline $\mathrm{E} 3-\mathrm{E} 4$ & $0,65-0,496$ & \\
\hline Mortalidade pós-tratamento & $0-0,5$ & 19 \\
\hline c) Custos Anuais $(\mathrm{R} \$)^{*}$ & Inferior-superior & Base de cálculo * \\
\hline Investigação+Diagnóstico & $286,48-859,44$ & 572,96 \\
\hline Tratamento E0 & $1.842,88-5.528,65$ & $3.685,77$ \\
\hline Tratamento E1 & $2.374,3-7.122,3$ & $4.748,60$ \\
\hline Tratamento E2 & $3.546,5-10.639,5$ & $7.093,00$ \\
\hline Tratamento E3 & $3.444,63-10.333,89$ & $6.889,26$ \\
\hline Tratamento E4 & $2.718,24-8.154,83$ & $5.436,49$ \\
\hline Seguimento & $201,72-605,16$ & 403,44 \\
\hline \multicolumn{3}{|l|}{ d) Valores do Equipamentos (reais) ${ }^{* *}$} \\
\hline Mamógrafo Analógico & $160.000,00$ & \\
\hline Mamógrafo Digital & $850.000,00$ & \\
\hline Ressonância Magnética & $2.200 .000,00$ & \\
\hline
\end{tabular}

Fontes: Tabela de Procedimentos, Medicamentos e OPM do SUS/2007 Ministério da Saúde ${ }^{* *}$

Na Tabela 1 estão discriminados os parâmetros empregados no modelo: a) probabilidades de transição; b) efetividade da intervenção; c) custos com diagnóstico e custos com equipamentos.

Para a modelagem utilizou-se o software TreeAge Software Pro Suite, da TreeAge Software Inc. $\mathrm{O}$ desenvolvimento do modelo da cadeia de Markov para o câncer de mama seguiu três passos: 1) desenvolvimento do modelo teórico; 2) identificação dos dados a serem colocados no modelo; e 3 ) simulação da modelagem.

Utilizou-se o estadiamento $\mathrm{TNM}^{18}$ para categorizar os cincos estados da cadeia de Markov como fator prognóstico de sobrevida. Desta maneira, obteve-se a probabilidade da coorte iniciar o processo de modelagem em estados que correspondem a: a) sem evidências; b) E0; c) E1; d) E2; e) E3 e f) E4.

No modelo, foram criados três estados absorvedores, dos quais os indivíduos não poderiam mais sair; a saber: a) morte por outras causas; b) sem evidencias pós-tratamento; e c) morte por câncer de mama. Após a realização do protocolo de tratamento, houve o seguimento anual das mulheres até o quinto ciclo markoviano. A partir deste momento, as mulheres passam a um estado absorvedor chamado de "sem evidências pós-tratamento". 


\section{Resultados}

Ao colocar as estratégias analisadas em ordem crescente de efetividade, observamos que o rastreamento com a ressonância magnética é mais efetivo, mas de custo mais elevado,

Uma análise de custo-efetividade das estratégias do rastreamento mamográfico, tendo como baseline o não rastreamento, gerou os resultados elencados na Tabela 2, onde os valores estão dispostos sem a taxa de desconto. Observou-se que a estratégia do rastreamento que apresentou melhor relação de custo por ano de vida ganhos é a mamografia convencional, com um custo igual a $\mathrm{R} \$ 74.627,00$ e 34,933 anos de vida ganhos.

A opção de menor custo é representada pelo não rastreamento, através da historia natural da doença. Em uma análise da razão CEI, as estratégias são avaliadas com base em seus custos e resultados. Após um exame mais detalhado, retira-se aquela estratégia de custo maior e menor efetividade. No caso em questão, a estratégia representada pelo rastreamento com a mamografia digital é dominada pelas demais. A Tabela 3 reorganiza os resultados obtidos com base na remoção da estratégia dita dominada, gerando um novo cálculo de CEI. Quando se avalia uma análise de custo-efetividade entre duas estratégias não dominadas, a Razão de Custo Efetividade Incremental (ICER) deve ser calculado. A menor ICER corresponde a um melhor valor, isto é, um menor custo por efetividade adicional.

A partir dos dados obtidos, observou-se que entre as estratégias de rastreamento modeladas, a ressonância magnética obteve a maior efetividade, com 34.949 anos de vida ganhos. A diferença dos custos entre a utilização das duas tecnologias, mamografia convencional e ressonância magnética, após a retirada da estratégia que foi dominada, chega a 46 milhões de reais na coorte analisada.

Em relação à ICER, em termos de custo por ano de vida ganhos, o rastreamento mamográfico convencional produziu um ano extra por R\$ 13.573,07, enquanto a ressonância magnética produziu um ano extra de vida por R\$ 2.904.328,88.

Realizou-se uma análise de sensibilidade a fim de avaliar o impacto e a robustez dos resultados.

Tabela 2. Razão incremental de custo-efetividade do rastreamento mamográfico com filme convencional, digital e ressonância magnética.

\begin{tabular}{lllllll}
\hline \multicolumn{1}{c}{ Cenários } & Custo (R\$) & $\begin{array}{c}\text { Custo } \\
\text { Incremental } \\
(\mathbf{R} \$)\end{array}$ & $\begin{array}{c}\text { Efetividade } \\
\text { (Anos) }\end{array}$ & $\begin{array}{c}\text { Efetividade } \\
\text { Incremental } \\
\text { (Anos) }\end{array}$ & $\begin{array}{c}\text { C/E } \\
\text { (R\$/Ano) }\end{array}$ & $\begin{array}{c}\text { Custo } \\
\text { Efetividade } \\
\text { Incremental } \\
\text { (ICER) }\end{array}$ \\
\hline $\begin{array}{l}\text { História Natural } \\
\text { do Câncer de Mama }\end{array}$ & $194.216,68$ & & 34,756 & & $5.588,00$ & \\
$\begin{array}{l}\text { Mamografia } \\
\text { convencional }\end{array}$ & $2.606 .986,65$ & $2.412 .769,97$ & 34,933 & 0,178 & $74.627,00$ & $13.573,072$ \\
$\begin{array}{l}\text { Mamografia digital } \\
\text { RessonânciaMagnética }\end{array}$ & $6.082 .348,27$ & $3.475 .361,61$ & 34,931 & $-0,003$ & $174.127,00$ & (Dominada) \\
\hline
\end{tabular}

Tabela 3. Razão de custo efetividade incremental sem as opções dominadas (simples ou estendida).

\begin{tabular}{|c|c|c|c|c|c|c|}
\hline Cenários & Custo (R\$) & $\begin{array}{c}\text { Custo } \\
\text { Incremental } \\
(\mathrm{R} \$)\end{array}$ & $\begin{array}{l}\text { Efetividade } \\
\text { (Anos) }\end{array}$ & $\begin{array}{c}\text { Efetividade } \\
\text { Incremental } \\
\text { (Anos) }\end{array}$ & $\begin{array}{c}\mathrm{C} / \mathrm{E} \\
(\mathrm{R} \$ / \text { Ano })\end{array}$ & $\begin{array}{c}\text { Custo } \\
\text { Efetividade } \\
\text { Incremental } \\
\text { (ICER) }\end{array}$ \\
\hline História Natural & $194.216,68$ & & 34,756 & & $5.588,00$ & \\
\hline $\begin{array}{l}\text { Mamografia } \\
\text { convencional }\end{array}$ & $2.606 .986,65$ & $2.412 .769,97$ & 34,933 & 0,178 & $74.627,00$ & $13.573,07$ \\
\hline Ressonância & $48.614 .388,31$ & $46.007 .401,6$ & 34,949 & 0,016 & $1.390 .998,00$ & $2.904 .328,88$ \\
\hline
\end{tabular}


Nas estratégias modeladas, o preço dos equipamentos tem um impacto maior nos custos nos diferentes cenários. Considerando que a mamografia convencional foi mais custo-efetiva, acrescentou-se $50 \%$ no valor do equipamento em um novo modelo. Não houve modificações nos resultados obtidos inicialmente, mesmo havendo variação no preço da tecnologia mais custo efetiva.

\section{Discussão}

Os estudos de custo-efetividade sobre o rastreamento mamográfico encontrados na literatura científica têm vários enfoques e metodologias diferentes ${ }^{5,7,10,19}$. Existem variações na estratégia e no enfoque do rastreamento, entre elas: a) variação do intervalo do rastreamento; b) faixa etária a ser rastreada; c) mulheres de grupo de risco; d) qualidade de vida ajustada a anos de vidas ganhos. Estas variáveis aumentam as controvérsias já existentes, mas também ajudam na descoberta de novas terapias além de diminuir custos ${ }^{19}$.

Considerando que um dos objetivos do presente trabalho é agregar subsídios para o processo de decisão de compra de novas tecnologias de rastreamento do câncer de mama pelo sistema público de saúde brasileiro, foram revisados alguns estudos, citados em seguida.

Em 1994, Rosenquist e Lindfors ${ }^{20}$ realizaram um estudo de custo efetividade do rastreamento mamográfico em mulheres na faixa etária de 40 49 anos, utilizando a Modelagem de Markov, empregando como medida de efetividade desta análise econômica os Anos de Vida Salvos. Os resultados demonstraram que, para esta faixa etária, o custo incremental por ano de vida salvo foi de US\$26.200 e US\$14.000 para o rastreamento anual e bianual, respectivamente; assumindo uma redução de 30\% na mortalidade. Quando reduziu-se em $20 \%$ a mortalidade, estes valores passaram a US $\$ 44.000$ e US\$ 23.000 por ano de vida salvo para o rastreamento anual e bianual, respectivamente. O principal problema encontrado no estudo foi a inexistência de ensaios clínicos randomizados que demonstrassem uma significante redução na mortalidade, na faixa etária de 40-49 anos.

Já Plans et al..$^{21}$, encontraram uma razão de custo efetividade por ano de vida salva de cerca de US\$ 7.020. O Sistema Público de Saúde do Reino Unido (National Health Service - NHS) publicou alguns artigos sobre analise de custo efetividade com vários enfoques. Em 2001, o NHS realizou uma análise de custo-efetividade do ras- treamento mamográfico em 160 mil mulheres na faixa etária de 50 a 69 anos, comparando com o não rastreamento na Noruega, com a perspectiva de custos do Sistema de Saúde local. Os benefícios mostraram um total de 41.279 anos de vida salvos nesta coorte. O custo por ano de vida salvo foi de \$3.750. Em 2005, outro estudo apoiado pelo NHS, avaliou a incorporação da imagem digital mamográfica no rastreamento de câncer de mama. Os resultados mostraram que, apesar do aumento da sensibilidade e especificidade da imagem digital, não houve grande diferença quando comparada à imagem de filme. Os autores concluíram que é mais custo efetivo o uso da mamografia convencional no rastreamento de câncer de mama ${ }^{21}$.

Uma limitação deste estudo é que, devido à não uniformização dos procedimentos para a realização dos laudos de interpretação da imagem, ao funcionamento inadequado dos equipamentos e câmaras escuras para filmes mamográficos, à técnica inadequada na utilização dos equipamentos mamográficos, à manutenção ineficiente, entre outras questões, a qualidade dos laudos fica bastante prejudicada em nosso país. Tais fatos diminuem a acurácia do exame e, consequentemente, há um aumento dos custos de diagnósticos e tratamento do câncer de mama.

\section{Conclusão}

A simulação mostrou que realizar um rastreamento mamográfico com o filme convencional, é mais custo efetivo do que as outras duas estratégias com outros equipamentos, na faixa etária de interesse.

Apesar da efetividade do rastreamento com IRM ser maior do que das demais alternativas, o preço do equipamento e os resultados falsos positivos elevaram consideravelmente os custos finais.

Os resultados foram baseados no rastreamento mamográfico bi-anual, numa coorte de 100 mil mulheres, faixa etária dos 50 aos 69 anos de idade, de acordo com o protocolo do INCA ${ }^{3}$.

A simulação computacional foi baseada em dados retirados da literatura, e algumas estimativas foram realizadas devido à falta de informações disponíveis, vide Tabela 1.

É importante ressaltar que modelagens computacionais que estimem resultados em longo prazo podem ter risco de imperfeições, devido à quantidade de eventos significativos que podem acontecer no futuro e que poderão alterar alguns dados ou fenômenos modelados. Como exem- 
plo, podemos citar a incorporação de novas tecnologias de rastreamento, o aumento da acurácia dos testes diagnósticos, o aumento da efetividade do tratamento existente ou a descoberta de novas terapias, além da diminuição dos custos.

Outra dificuldade encontrada foi a inexistência de estudos relacionados ao tema, na literatura brasileira, a fim de que pudessem ser realizadas comparações nacionais ${ }^{22}$.

A importância da realização deste trabalho foi a possibilidade de se analisar o impacto das tecnologias de rastreamento mamográfico em mulheres brasileiras, na perspectiva do Sistema Único de Saúde. As informações são relevantes para os tomadores de decisão, possibilitando intervenções com mais efetividade na alocação de recursos, inclusive com possibilidades de estudo dos limiares desta intervenção, nas faixas etária de interesse, o que não seria possível em situações reais.

\section{Colaboradores}

AAF Peregrino, CEV Almeida, CMM Vianna, FVC Silva, GB Gonzalez, MPS Rodrigues e SCF Machado participaram igualmente de todas as etapas de elaboração do artigo. 
Referências

1. Organização Mundial da Saúde (OMS). União Internacional de Combate ao Câncer. Acción Mundial Contra el Cancer. Genebra. [Internet]. 2006 [acessado 2007 mar 24]. Disponível em: <http://www. uicc.org/fileadmin/about/accion.pdf $>$

2. Instituto Nacional de Câncer (Inca). Estimativas 2010. Incidencia e Mortalidade de Câncer no Brasil. Rio de Janeiro: CONPREV, Instituto Nacional de Câncer (Inca); 2009.

3. Instituto Nacional de Câncer (Inca). Falando sobre o Câncer de Mama: Programa Nacional do Câncer do Colo de Útero e de Mama. Rio de Janeiro: CONPREV, Inca; 2000.

4. America College of Radiology. The ACR Breast Imaging Reporting and Data System. [Internet] 2005 [acessado 2005 jun 13]. Disponível em: http://www. acr.org./s_acr/bin.asp?

5. Nass SJ, Henderson C, Lashof JC. Mammography and Beyond: Developing Technologies for the Early Detection of Breast Cancer. Washington: National Research Council; 2001.

6. Johnston K. Modeling the future cost of breast screening. Eur J Cancer 2001; 37(14):1752-1758.

7. Hider P, Nicholas B. The Early Detection and Diagnosis of Breast Cancer: a literature review - an update. NZHTA Report 1999; 2(2)

8. Chen HH, Thurfjell E, Duffy SW, Tabar L. Evalua tion by Markov Chain Models of a Non-randomised Breast Cancer Screening Programme in Women aged under 50 years in Sweden. J Epidemiol Community Health 1998; 52(5):329-335.

9. Gold RH, Basset LW, Widoff BE. Highlights from the History of Mammography. Los Angeles. Radiographics 1990; 10(6):1111-1131.

10. Salzman P, Kerlikowske K, Philips K. Cost-Effectiveness of Extending Screening Mammography Guidelines to Include Women 40 to 49 Years of Age. Ann Intern Med 1997; 127(11):955-965.

11. Rafferty EA. Digital Mammmography: Novel Applications. Radiol Clin North Am 2007; 45(5):831-843.

12. Freitas AG, Kemp C, Louveira MH, Fujiwara SM Campos LF. Mamografia Digital: Perspectiva atual e aplicações futuras. Radiol Bras 2006; 39(4):287-296.

13. Nees A. Digital Mammography: Are There Advantages in Screening for Breast Cancer? Acad Radiol 2008;15(4):401-407.
14. Pisano ED, Gatsonis C, Hendrick E, Yaffe M, Baum JK, Acharyya S, Conant EF, Fajardo LL, Bassett L, D’Orsi C, Jong R, Rebner M; Digital Mammographic Imaging Screening Trial (DMIST) Investigators Group. Diagnostic Performance of Digital versus Film Mammography for Breast-cancer screening. N Engl J Med 2005; 353(17):1773-1783.

15. DeMartin W, Lehman C, Partridgte S. Breast MRI for cancer detection and caharacterization: A Review of Evidence-Based Clinical Applictions. Acad Radiol 2008; 15(4):408-416.

16. Nemec CF, Listinsky J, Rim A. How should we screen for breast câncer? Mammography, ultrasonography, MRI. Cleve Clin J Med. 2007; 74(12):897-904.

17. Hunter DJ, Drake SM, Shortt SE, Dorland JL, Tran N. Simulation modeling of change to breast cancer detection age eligibility recommendations in Ontario, 2002-2021. Cancer Detect Prev 2004; 28(6):453-460.

18. Instituto Nacional de Câncer (Inca). Classificação TNM: Classificação de Tumores Malignos 6a ed [Internet] 2004 [acessado 2009 nov 12]. Disponível em: www.inca.gov.br/tratamento/tnm/tnm2.pdf

19. Instituto Nacional de Câncer (Inca). Coordenação de Prevenção e Vigilância. Controle de Câncer de Mama: Documento de Consenso. Rio de Janeiro: CON PREV, Instituto Nacional do Câncer (Inca); 2004

20. Rosenquist CJ, Lindfors KK. Screening mammography in women aged 40-49 years: analysis of cost effectiveness. Radiology. 1994; 191(3):647-650.

21. Plans P, Casademont L, Salleras L. Cost-effectiveness of breast cancer screening in Spain. Int J Technol Assess Health Care 1996; 12(1):146-150.

22. National Health Service (NHS). Health Information Resources: Formerly National Library for Health. [Internet] 2010 [acessado 2010 jan 13]. Disponível em: http://www.library.nhs.uk/default.aspx

Artigo apresentado em 25/10/2009

Aprovado em 21/02/2010

Versão final apresentada em 15/03/2010 